\title{
Cultural Adaptation of Health Literacy Measures: Translation Validation of the Newest Vital Sign in Arabic-speaking Parents of Children with Type 1 Diabetes in Kuwait.
}

Dalia Al-Abdulrazzaq ( $\nabla$ d.alabdulrazzaq@hsc.edu.kw)

Kuwait University https://orcid.org/0000-0002-4613-865X

Abdullah Al-Taiar

School of Community and Environmental Health

Muneera Al-Haddad

Ministry of Health

Abeer Al-Tararwa

Ministry of Health

Nabeela Al-Zanati

Ministry of Health

Amna Al-Yousef

Ministry of Health

Lena Davidsson

Dasman Diabetes Institute

Hessa Al-Kandari

Dasman Diabetes Institute

Research article

Keywords: health literacy, newest vital sign, Arabic, adaptation, Kuwait

Posted Date: January 6th, 2020

DOI: https://doi.org/10.21203/rs.2.20083/v1

License: (c) (i) This work is licensed under a Creative Commons Attribution 4.0 International License.

Read Full License

Version of Record: A version of this preprint was published at The Science of Diabetes Self-Management and Care on February 28th, 2021. See the published version at 
https://doi.org/10.1177/0145721721996309. 


\section{Abstract}

\section{Background}

Low level of health literacy $(\mathrm{HL})$ has been linked to several adverse health outcomes independently from education, ethnicity and socio-economic status. There is a dire need for a reliable tool to assess $\mathrm{HL}$ of patients and general public in Arab countries. This study aimed to assess the feasibility of use and reliability of the Arabic version of the Newest Vital Sign (NVS) in parents of children with type 1 diabetes (T1D).

\section{Methods}

Translation and cross-cultural adaptation of the Arabic version of the NVS (NVS-Ar) was conducted according to established guidelines. In order to investigate the functionality of NVS, the final version of NVS-Ar was administered to 175 adult caregivers of children with T1D, who are native Arabic speakers. We assessed the association between NVS-Ar score for the parents and HbA1C for their children. The internal consistency was evaluated by Cronbach's Alpha (CA) and reliability was assessed by test retest method.

Results

The median (interquartile rang IQR) score was 4.0 (3-5). The internal consistency of the NVS-Ar was moderate $(C A=0.58)$. While the intraclass correlation coefficient (ICC) was 0.61 . There was no correlation between NVS-Ar score and HbA1C (Spearman's rho $=0.055 ; \mathrm{p}=0.62$ ). Furthermore, there was significant inverse association between adequate $\mathrm{HL}$ and optimal glycemic control among children, which remained evident even after adjusting for the duration of T1D, age or education of the parents/child guardian. However, it lost statistical significance when we adjust for treatment regimen.

\section{Conclusion}

Our findings demonstrate that NVS is unlikely to be a predictive tool for functional HL in Arabic settings and that there is a need to properly translate and validate other tools such as TOFLA or alternatively develop a reliable tool de novo.

\section{Introduction}

Health Literacy $(\mathrm{HL})$ is defined as "the cognitive and social skills that determine the motivation and ability of individuals to gain access to, understand and use information in ways that promote and maintain good health" [1, 2]. It is a complex concept with particular focus on the ability to process and understand both linguistic and numeric information in a health context. Such ability is critical to make appropriate informed health-related decisions. Inadequate $\mathrm{HL}$ has been linked to adverse health independently from education, ethnicity and socio-economic status [3]. As an example, inadequate HL was linked to smoking [4], hospitalization [5], poor glycemic control in diabetic patients [3, 6, 7], feeding formula milk for infants 
[8], limited participation in screening for diseases [9-12], self-reported poor health [13] and an increase in all-cause mortality [14-16].

In clinical settings, it is envisaged that when there is a gap between patients' HL and their demands of the healthcare system, those with inadequate $\mathrm{HL}$ are particularly disadvantaged [17]. As part of clinical practice, patients are given written and verbal information about their medical conditions, treatment or preventive procedures; and are asked to sign different types of forms and consent to medical procedures. Patients with inadequate $\mathrm{HL}$ might have difficulties in understanding complex information and can therefore face difficulties when making informed decisions regarding treatment or other matters related to their medical conditions. Treating physicians need to have knowledge on the HL level within their patient population in order to provide information at an appropriate level to help patients make fully informed decisions regarding their healthcare [18]. For example, pooled prevalence data shows that nearly one in three patients with type 2 diabetes in the US have limited HL [19]. Unfortunately, physicians often fail to identify individual patients with limited $\mathrm{HL}[20,21]$ Clearly, patients with limited language skills, illiterate or semi-literate will be disadvantaged in $\mathrm{HL}$ testing and it is thus important to note that most patients are unwilling to admit that they have literacy problems [5].

An appropriate and objective measure of $\mathrm{HL}$ is crucial to investigate the impact of inadequate $\mathrm{HL}$ on individuals' health and healthcare use in different cultures. The test of functional health literacy in adults (TOFHLA) and the rapid estimate of adult literacy in medicine (REALM) are two of the most commonly used tools to measure $\mathrm{HL}[22,23]$. However, these tools have some limitations to their usefulness in different settings. For example, the TOFHLA tool takes around 22 minutes to be completed [22] and is therefore not practical for use in a busy clinical setting. Furthermore, many of these tools have not been translated and validated in major languages such as Arabic with distinct dialects spoken in different countries. The short version of the Functional Health Literacy Test for Adults (S-TOFHLA) has been translated to Arabic but no further steps to validate the tool have been made [24]. The S-TOFHLA and the Rapid estimate of adult literacy in medicine Revised (REALM-R) have been translated to Arabic for use in the Lebanese population only [25].

In 2005, Weiss et al [26] developed a screening instrument, the Newest Vital Sign (NVS), to assess an individual's level of $\mathrm{HL}$. In an average of 3 minutes, the NVS assesses mathematics, reading, comprehension skills, and abstract reasoning based on six questions referring to an ice cream nutrition label. This rapid test has been found to be as sensitive in identifying those with inadequate $\mathrm{HL}$ as the gold standard measure of HL namely TOFHLA [22]. The NVS has been widely used widely in the US and has been validated for use in the UK [17]. The test has been translated to other languages, for example Dutch [27], Turkish [28] and Japanese [29] but has been only translated to Arabic with no further validation [24].

In Arab countries, $\mathrm{HL}$ has been adopted by the United Nations as an important sustainable developmental goal [30], yet it remains a neglected topic. It is crucial to have a properly validated tool to assess HL in Arabic-speaking populations. The lack of a valid tool to measure HL in Arabic settings has become a 
hindrance to address $\mathrm{HL}$ and its impact on health status and healthcare utilization in the Arab world. As a striking example of the dire need for addressing $\mathrm{HL}$ in Arab countries, Al-Taiar et al reported that the majority of patients on the waiting list for knee replacement surgery were unaware of the benefit of the procedure or the time the prosthesis will last and attributed the delay in taking the procedure to the lack of information [31]. Similarly, more than $60 \%$ of type 2 diabetes patients showed inadequate or marginal HL using TOFHLA [32].

This study aimed (a) to translate and validate the NVS tool into Arabic and (b) to assess the feasibility of use and reliability of the Arabic version of the NVS in parents of children with type 1 diabetes (T1D).

\section{Methods}

\section{Phase 1: Translation of the English version of the NVS instrument to Arabic (Figure.1, Panel A)}

Translation and cross-cultural adaptation of the Arabic version of the NVS (NVS-Ar) was conducted according to established guidelines [33,34]. First phase included forward-translation, expert panel review, and backward-translation of the original English version of the NVS instrument (Form A). The original instrument, including the food label and the questions, were translated into Arabic independently by two bilingual translators. Both translators were instructed to compare the two separate Arabic translations in order to produce a single reconciled Arabic version of the instrument (Form B). An expert panel was formulated to review the Arabic version of the NVS instrument from the linguistic and cultural perspectives. They made changes to the food label translation to be in line with the food labeling system used in Kuwait namely the translation of "serving size", "serving per container" and "amount per serving". The numeric values were kept in the English language in the food label as per the labeling system in the country. The expert panel consisted of physicians, clinical nurses, dieticians, and local food manufacturers. After the review and modification, the final Arabic version (Form C) was back-translated to English (Form D) independently by two different bilingual translators who were not familiar with the original English instrument. As for the forward-translation, the translators compared their two backtranslated versions and produced a single reconciled English version of the instrument.

\section{Phase 2: Translation validation of the Arabic version of the NVS-Ar instrument (Figure.1, Panel B)}

In order to validate the translation from the first phase, we followed the methodology described in the guidelines $[33,35,36]$. This method involves formal comparison of the original English NVS (Form A) and the back-translated to English (Form D). Medical students $(n=11)$ faculty and support staff $(n=12)$ at the Faculty of Medicine, Kuwait University compared each item in the two English versions and ranked them as to comparability of language and similarity of interpretation using a 7-point Likert-type scale (Comparability of language, $1=$ Extremely comparable and $6=$ Not at all comparable; Similarity of interpretation, $1=$ Extremely similar and $6=$ Not all similar). Medical students and staff at the Faculty of Medicine are fluent in English. Comparability of language refers to the formal similarity of words, phrases, and sentences while similarity of the interpretation refers to the degree to which the two versions would engender the same attitude response even if the wording were not the same. Items with mean 
scores above 3 for comparability and/or similarity were re-evaluated. This step is aimed at identifying problematic items and retranslate them till an item is interpreted in the same manner in both languages (Figure 1, Panel B). Findings from this phase are shown in Table 1. The final version of the Arabic version of the NVS-Ar is provided in Appendix 1.

\section{Phase 3: Feasibility and reliability testing}

Testing was conducted in a group of parents or legal guardians of children with Type 1 diabetes (T1D) attending hospital-based out-patient diabetes clinics in Kuwait. The final Arabic version of NVS-Ar was administered to 175 native Arabic speaking parents/guardians. Caregivers who had documented learning disability or self-reported visual abnormalities preventing them from visualizing the food label were excluded from the study. A subgroup of participants was asked to revisit the hospital and a re-test was done 1-3 weeks later.

\section{Phase 4: Validity and functionality testing}

Previous studies have shown that $\mathrm{HL}$ assessed in parents related to glycemic control of their children [37]. Therefore, we investigated the functionality of NVS-Ar by assessing the association between the NVS-Ar score of the parent/guardian with their child's hemoglobin A1C(HbA1C). Optimal glycemic control in this study was defined as $\mathrm{HbA1C}$ of $\otimes 7.5 \%$ as per the 2018 International Society for Pediatric and Adolescent Diabetes (ISPAD) [38].We also assessed the validity of NVS-Ar by investigating the association between NVS-Ar score and educational achievement.

\section{Data analysis}

Data were analyzed using Stata 12. (Stata Corporation, College Station, TX, USA). Categorical variables were summarized by proportions while quantitative variables by mean (standard deviation-SD) or median (interquartile range-IQR). The feasibility of NVS-Ar was assessed by analyzing the number of questions answered and the time that takes the participants to do the test. Internal reliability was assessed by Cronbach alpha (CA). External reliability was assessed using a test-retest method using intraclass correlation coefficient (ICC) with two-way mixed effects model.

We assessed the validation NVS-Ar by calculating the association with educational achievement. We also investigated the functionality of NVS-Ar by assessing the correlation between NVS-Ar score for the parents and $\mathrm{HbA} 1 \mathrm{C}$ for their children. Logistic regression was used to investigate the association between NVS-Ar score (as a continuous and categorical variable) and the $\mathrm{HbA} 1 \mathrm{C}$ while adjusting for potential confounders.

\section{Results}

\section{Translation validation of the Arabic version of the NVS instrument}


In order to validate the translation, medical students, faculty and supportive staff at the Faculty of Medicine, Kuwait University systematically compared the original English NVS and the back-translated to English ((Form A and Form D in Figure 1). Table 1 shows the median comparability and similarity scores of each item on the questions/scoring sheet of the NVS instrument. Back-ward translation produced an identical wording for Question 6, and therefore, was not included in the comparability and similarity testing. Two items were considered problematic during this phase and had to be re-translated and reevaluated till the desired mean scores were obtained (Figure 1, Panel B).

\section{Feasibility and Reliability testing}

The final Arabic version of the NVS-Ar instrument was administered to 175 participants. Table 2 shows the socio-demographic characteristics of the parents or child guardian who participated in the study. The majority of the study participants were Kuwaiti 108 (62.79\%) and predominantly mothers 132 (75.86). The mean (SD) age of the index child was 9.20 (2.98) years. The median (IQR) of the duration of T1D was 32.37 (18.97-54.93) months.

The final Arabic version of NVS consists of a nutritional label and six questions, with one point awarded for each correct answer, giving a minimum score of 0 and a maximum score of 6 . The distribution of the NVS-Ar score is shown in Figure 2. The distribution is negatively skewed with large number of participants scoring higher score which indicate good level of HL. The median (IQR) score was 4.0 (3-5). Difficulty of individual questions are shown in table 3. Only six (3.43\%) participants scored zero; and the median (IQR) of the time required to do the test was 4.36 (3-7) minutes. The internal consistency of the NVS-Ar was moderate (CA 0.58). While the ICC as a measure of a test retest reliability was 0.61 .

\section{Assessing the validity of NVS-Ar}

We assessed the validity of NVS-Ar by investigation the association between NVS-Ar score and educational level. Table 4 show the association between NVS-Ar score and the educational level. There was no association between NVS-Ar score and education level $(p=0.423)$. This remain unchanged after adjusting for age, gender and nationality. Because, previous studies showed HL is related to glycemic control of their children [37], we investigated the functionality of NVS-Ar by assessing the association between NVS-Ar score for the parents and $\mathrm{HbA1C}$ for their children. There was no correlation between NVS-Ar score and HbA1C (Spearman's rho $=0.055 ; p=0.62$ ). Furthermore, there was significant inverse association between adequate $\mathrm{HL}$ (score $>3$ ) and optimal glycemic control among children (those with adequate $\mathrm{HL}$ tended to have children with higher $\mathrm{HbA1C}$ ) (Figure.3). This remain evident even after adjusting for the duration of T1D and age or education of the parents/child guardian. However, it lost statistical significance when we adjust for treatment regimen. It is worth noting that educational level showed positive association with proper glycemic control although it did not reach statistical significance.

\section{Discussion}


It is increasingly recognized that inadequate $\mathrm{HL}$ is associated with poor health-related knowledge and comprehension and as a result adverse health-outcomes [39]. Mounting evidence now supports a growing awareness that general $\mathrm{HL}$ is a major individual factor affecting an individual's health status [40]. The availability of reliable instruments to measure $\mathrm{HL}$ has contributed to the raising awareness on the impact of HL on individuals' health [22, 26, 41, 42]. Most of the instruments were developed and validated in English and, because it is difficult to develop and validate new instruments in other languages de novo, such instruments are translated to other languages to be adopted in different cultures. However, it is a challenge to adapt such instruments in a culturally relevant and comprehensible form while maintaining the meaning and the intent of the original items [33].

In this study we report on the translation and validation of the NVS instrument into Arabic using a validation process as described in international guidelines $[33,35,36]$. CA was 0.58 , which is not high as an index of internal consistency of a test. However, CA is only a reflection of the inter-relatedness of the items within the test [43], (if the items in a test are correlated to each other, the value of CA is increased) and because the fifth and sixth questions are meant to measure comprehension while the first four questions (questions 1 to 4) meant to measure numerical skills such low CA is not surprising. Furthermore, CA is affected by the length of the test and if the test is too short like NVS, the value of CA is reduced. In fact, a high level of CA has never been reported from any study. CA was reported to be 0.69 among 85 participants in Iraq [24], 0.70 in Turkey [28], 0.74 in UK [17], 0.69 in Spain [26] and 0.76 for NVS$D$ in Netherlands [27]. Although CA of $\geq 0.70$ is deemed to be satisfactory [44], there is numerous criticism for this approach [43]. A test with $C A=0.70$ still has huge amount of error that may exceeds $50 \%$ of the results of the test.

We agree with the previous studies that the fifth question "is it safe for you to eat this ice cream?" is particularly problematic [45]. This question requires a dichotomous answer "yes, no" which can be easily answered by guessing. Salgado et al [45] showed that most of those who answered the question correctly failed to report the reason for their answer, which is requested in the next question. In our study, the fifth question was most often answered correctly but approximately one third of those who answered the fifth question correctly failed to explain why in the next question. In Turkey the fifth question was the most correctly answered question [28]. As mentioned above, unlike the first four questions, fifth and sixth question meant to evaluate comprehension, and this also undermine the reliability NVS.

Most of the previous studies did not report test retest validity (external reliability). We assessed external reliability using a test-retest method using intraclass correlation coefficient (ICC) with two-way mixed effects model. This was found to be 0.61 , a number that is deemed to be low. Usually, intra-class correlation coefficient of $>0.80$ is indicative for excellent reliability [46].

We found no association between NVS- Ar Score and the educational level of the parents/guardians. This finding is in agreement with previous studies which showed no link between NVS score and educational level $[17,24,45]$ although some studies have reported a link [28]. In UK, NVS score was reported to have a weak correlation with educational attainment [17]. Nevertheless, it is known that educational 
achievements are not good predictor of literacy skills as many individuals have literacy skills well below what might be expected from their level of education [17].

Previous studies showed that $\mathrm{HL}$ of the parents of children with diabetes is related to glycemic control of their children [37], thus we investigated the functionality of NVS-Ar by assessing the association between NVS-Ar score for the parents/guardians and $\mathrm{Hb} 1 \mathrm{AC}$ for their children with T1D. Our results show no correlation between NVS-Ar score and the HbA1C of the diabetic children. In fact, there was a significant inverse association between HL of the parents as measured by NVS-Ar (Score $\geq 4$ ) and optimal glycemic control in their children. Our results are not surprising as NVS previously was shown to have a limited utility in predicting medication adherence among Portuguese adult [45] although this was attributed to floor effect (large number of participants scored very low score). In our study, despite the average high score (median 4.0) (i.e. limited floor effect), the NVS-Ar showed limited predictability of HbA1C which cast doubt on the predictive value of the NVS-Ar in our setting. Al-Jumaili et al [24] suggested that NVS might not be applicable as HL test for people in Iraq who are not accustomed to reading product labels in their daily life. In the Netherlands, NVS was found to be problematic and a new NVS for Dutch people was developed [27].

NVS is attractive because of its brevity and covering both reading and numerical skills. However, the drawbacks of NVS include low reliability and doubts about its validity. The validity of NVS has been investigated by running a parallel HL test such as TOFLA which does not really show the functionality of the tool. Our findings suggest that NVS is too short to provide reliable results and to be a predictive tool for functional $\mathrm{HL}$ at least in our setting.

$\mathrm{HL}$ is an increasingly researched area in health care in the West compared to a severe lack of such research in the Arab world. Our current study highlights the need for the future research and international comparison of $\mathrm{HL}$ in Arabic-speaking populations. It presents a validated and rigorous cross-cultural adaptation process of the measurement tool that addressed potential differences in cultural interpretation of language and its utilization. However, there are some limitations to the present study. Firstly, due to the lack of a "gold standard" Arabic tool to test $\mathrm{HL}$ among adults, the process of validating the Arabic version of the NVS is incomplete. Secondly, to test feasibility and reliability of the Arabic version, adult caregivers of children under follow-up at the Diabetes clinics were selected. These participants may have different HL skills compared to the general adult population in the country which might limit the generalizability of our findings. Testing of the Arabic version of the NVS on other adult populations could further validate the feasibility and reliability of the instrument.

In conclusion, our findings demonstrate that NVS is unlikely to be a predictive tool for functional HL in Arabic settings and that there is a need to properly translate and validate other tools such as TOFLA or alternatively develop a reliable tool de novo. Such work is a prerequisite for initiatives that aim to improve $\mathrm{HL}$ in Arab countries.

\section{Abbreviations}


HL: Health literacy

TOFHLA: test of functional health literacy in adults

REALM: the rapid estimate of adult literacy in medicine

S-TOFHLA: The short version of the Functional Health Literacy Test for Adults

REALM-R: The Rapid estimate of adult literacy in medicine Revised

NVS: Newest vital sign

T1D: Type 1 diabetes

NVS-Ar: Arabic version of the NVS

HbA1C: hemoglobinA1C

ISPAD: International Society for Pediatric and Adolescent Diabetes

SD: Standard deviations

IQR: Interquartile range

CA: Cronbach alpha

ICC: intraclass correlation coefficient

\section{Declarations}

\section{Ethics approval and consent to participate:}

This study was reviewed and approved by the Joint Committee for The Protection of Human Subjects in Research of the Health Sciences Centre and Kuwait Institute of Medical Specialization. Consent was obtained verbally from participating subjects.

\section{Consent for publication}

Approved by the Joint Committee for The Protection of Human Subjects in Research of the Health Sciences Centre and Kuwait Institute of Medical Specialization.

\section{Availability of data and materials}

The datasets generated and/or analysed during the current study are not publicly available because the Joint Committee for The Protection of Human Subjects in Research of the Health Sciences Centre and 
Kuwait Institute of Medical Specialization did not approve of sharing these datasets but are available from the corresponding author on reasonable request.

\section{Competing interests}

The authors declare that they have no competing interests.

\section{Funding}

This study has been funded by the University of Kuwait (Grant ZM04/12).

\section{Authors' contributions}

DA-A made the main contribution to the conception and design, acquisition of data, analysis and interpretation of data, and drafted the manuscript. AB-T1 had contributed to the analysis, interpretation of data and drafting of this manuscript. MU-A, AB-T2, NA-Z, AM-Y had contributed to the acquisition of data. LE-D and HA-K had contributed to the drafting and revision of the manuscript.

\section{Acknowledgements}

The authors gratefully acknowledge the children and their families who participated in the study.

\section{References}

1. Kickbusch, I.S., Health literacy: addressing the health and education divide. Health Promot Int, 2001. 16(3): p. 289-97.

2. Murphy, R., S. Ellard, and A.T. Hattersley, Clinical implications of a molecular genetic classification of monogenic beta-cell diabetes. Nat Clin Pract Endocrinol Metab, 2008. 4(4): p. 200-13.

3. Schillinger, D., et al., Association of health literacy with diabetes outcomes. JAMA, 2002. 288(4): p. 475-82.

4. Hawthorne, G., Preteenage drug use in Australia: the key predictors and school-based drug education. J Adolesc Health, 1997. 20(5): p. 384-95.

5. Safeer, R.S. and J. Keenan, Health literacy: the gap between physicians and patients. Am Fam Physician, 2005. 72(3): p. 463-8.

6. Tang, Y.H., et al., Health literacy, complication awareness, and diabetic control in patients with type 2 diabetes mellitus. J Adv Nurs, 2008. 62(1): p. 74-83.

7. Saeed, H., et al., Impact of health literacy on diabetes outcomes: a cross-sectional study from Lahore, Pakistan. Public Health, 2018. 156: p. 8-14.

8. Yin, H.S., et al., Parent health literacy and "obesogenic" feeding and physical activity-related infant care behaviors. J Pediatr, 2014. 164(3): p. 577-83 e1. 
9. Lindau, S.T., et al., The association of health literacy with cervical cancer prevention knowledge and health behaviors in a multiethnic cohort of women. Am J Obstet Gynecol, 2002. 186(5): p. 938-43.

10. Garbers, S. and M.A. Chiasson, Inadequate functional health literacy in Spanish as a barrier to cervical cancer screening among immigrant Latinas in New York City. Prev Chronic Dis, 2004. 1(4): p. A07.

11. Dolan, N.C., et al., Colorectal cancer screening knowledge, attitudes, and beliefs among veterans: does literacy make a difference? J Clin Oncol, 2004. 22(13): p. 2617-22.

12. Komenaka, I.K., et al., Association of Health Literacy With Adherence to Screening Mammography Guidelines. Obstet Gynecol, 2015.

13. Baker, D.W., et al., The relationship of patient reading ability to self-reported health and use of health services. Am J Public Health, 1997. 87(6): p. 1027-30.

14. Baker, D.W., et al., Health literacy and mortality among elderly persons. Arch Intern Med, 2007. 167(14): p. 1503-9.

15. Baker, D.W., et al., Health literacy, cognitive abilities, and mortality among elderly persons. J Gen Intern Med, 2008. 23(6): p. 723-6.

16. Bostock, S. and A. Steptoe, Association between low functional health literacy and mortality in older adults: longitudinal cohort study. BMJ, 2012. 344: p. e1602.

17. Rowlands, G., et al., Development and validation of a measure of health literacy in the UK: the newest vital sign. BMC Public Health, 2013. 13: p. 116.

18. Shah, L.C., et al., Health literacy instrument in family medicine: the "newest vital sign" ease of use and correlates. J Am Board Fam Med, 2010. 23(2): p. 195-203.

19. Abdullah, A., et al., Prevalence of limited health literacy among patients with type 2 diabetes mellitus: A systematic review. PLoS One, 2019. 14(5): p. e0216402.

20. Bass, P.F., 3rd, et al., Residents' ability to identify patients with poor literacy skills. Acad Med, 2002. 77(10): p. 1039-41.

21. Rogers, E.S., L.S. Wallace, and B.D. Weiss, Misperceptions of medical understanding in low-literacy patients: implications for cancer prevention. Cancer Control, 2006. 13(3): p. 225-9.

22. Parker, R.M., et al., The test of functional health literacy in adults: a new instrument for measuring patients' literacy skills. J Gen Intern Med, 1995. 10(10): p. 537-41.

23. Davis, T.C., et al., Rapid estimate of adult literacy in medicine: a shortened screening instrument. Fam Med, 1993. 25(6): p. 391-5.

24. Al-Jumaili, A.A., M.D. Al-Rekabi, and B. Sorofman, Evaluation of instruments to assess health literacy in Arabic language among Iraqis. Res Social Adm Pharm, 2015.

25. Fadda, M., et al., Validation of three Arabic health literacy assessment tools in Lebanon. Health Promot Int, 2018. 33(2): p. 261-267.

26. Weiss, B.D., et al., Quick assessment of literacy in primary care: the newest vital sign. Ann Fam Med, 2005. 3(6): p. 514-22. 
27. Fransen, M.P., et al., International application of health literacy measures: adaptation and validation of the newest vital sign in The Netherlands. Patient Educ Couns, 2014. 97(3): p. 403-9.

28. Ozdemir, H., et al., Health literacy among adults: a study from Turkey. Health Educ Res, 2010. 25(3): p. 464-77.

29. Kogure, T., et al., Validity and reliability of the Japanese version of the Newest Vital Sign: a preliminary study. PLoS One, 2014. 9(4): p. e94582.

30. Nationas, U. Sustainable development goals. 2019 [cited 2019 December 9]; Available from: https://www.un.org/sustainabledevelopment/sustainable-development-goals/.

31. Al-Taiar, A., et al., Attitudes to knee osteoarthritis and total knee replacement in Arab women: a qualitative study. BMC Res Notes, 2013. 6: p. 406.

32. Hussein, S.H., A. Almajran, and A.N. Albatineh, Prevalence of health literacy and its correlates among patients with type I/ diabetes in Kuwait: A population based study. Diabetes Res Clin Pract, 2018.

141: p. 118-125.

33. Sperber, A.D., Translation and validation of study instruments for cross-cultural research. Gastroenterology, 2004. 126(1 Suppl 1): p. S124-8.

34. Guillemin, F., C. Bombardier, and D. Beaton, Cross-cultural adaptation of health-related quality of life measures: literature review and proposed guidelines. J Clin Epidemiol, 1993. 46(12): p. 1417-32.

35. Sperber, A., R. DeVellis, and B. Boehlecke, Cross-cultural translation: methodology and validation. J Cross-Cult Psychol, 1994. 25: p. 501-524.

36. Wild, D., et al., Principles of Good Practice for the Translation and Cultural Adaptation Process for Patient-Reported Outcomes (PRO) Measures: report of the ISPOR Task Force for Translation and Cultural Adaptation. Value Health, 2005. 8(2): p. 94-104.

37. Hassan, K. and R.A. Heptulla, Glycemic control in pediatric type 1 diabetes: role of caregiver literacy. Pediatrics, 2010. 125(5): p. e1104-8.

38. DiMeglio, L.A., et al., ISPAD Clinical Practice Consensus Guidelines 2018: Glycemic control targets and glucose monitoring for children, adolescents, and young adults with diabetes. Pediatr Diabetes, 2018. 19 Suppl 27: p. 105-114.

39. Berkman, N.D., et al., Low health literacy and health outcomes: an updated systematic review. Ann Intern Med, 2011. 155(2): p. 97-107.

40. Scotten, M., Parental Health Literacy and Its Impact on Patient Care. Prim Care, 2015. 42(1): p. 1-16.

41. Davis, T.C., et al., Rapid assessment of literacy levels of adult primary care patients. Fam Med, 1991. 23(6): p. 433-5.

42. Davis, T.C., et al., Development and validation of the Rapid Estimate of Adolescent Literacy in Medicine (REALM-Teen): a tool to screen adolescents for below-grade reading in health care settings. Pediatrics, 2006. 118(6): p. e1707-14.

43. Tavakol, M. and R. Dennick, Making sense of Cronbach's alpha International Journal of Medical Education 2011. 2: p. 53-55. 
44. Nannally, J. and I. Bernstein, Psychometric theory. 1994, New York: McGraw-Hill Education

45. Salgado, T.M., et al., Newest Vital Sign as a proxy for medication adherence in older adults. J Am Pharm Assoc (2003), 2013. 53(6): p. 611-7.

46. Shrout, P.E. and J.L. Fleiss, Intraclass Correlations: Uses in Assessing Rater Reliability. Psychological Bulletin, 1979. 2: p. 420-428.

\section{Tables}

Table 1. Comparability and Similarly scores of the NVS questions.

\begin{tabular}{lc}
\hline Question & Median score (IQR) \\
\hline Question 1 & $2.0(1.0,3.0)$ \\
Comparability & $1.0(1.0,2.0)$ \\
\hline Similarity & $2.0(1.0,20.0)$ \\
Question 2 & $1.0(1.0,2.0)$ \\
\hline Comparability & \\
Similarity & $2.0(1.0,2.0)$ \\
Question 3 & $1.0(1.0,2.0)$ \\
\hline Comparability & \\
Similarity & $2.0(1.0,3.0)$ \\
Question 4 & $2.0(1.0,3.0)$ \\
\hline Comparability & $1.0(1.0,2.0)$ \\
\hline Similarity & $1.0(1.0,1.0)$ \\
\hline Question 5 & \\
\hline Comparability & Similarity \\
\hline
\end{tabular}

NVS: Newest vital sign, SD: Standard Deviation, SD: Standard Deviation.

Table 2. Socio-demographic characteristics of 175 parents/child guardians and their children with T1D. 


\begin{tabular}{|c|c|}
\hline Care givers of children with T1D & \\
\hline Age $^{a}$, years, mean (SD) & $38.27(6.24)$ \\
\hline $\begin{array}{l}\text { Relation to the index child }{ }^{\mathrm{b}}, \mathrm{n}(\%) \\
\text { Mother } \\
\text { Father } \\
\text { Other }\end{array}$ & $\begin{array}{c}132(75.86) \\
40(22.99) \\
2(1.14)\end{array}$ \\
\hline $\begin{array}{l}\text { Nationality }{ }^{c} \\
\text { Kuwaiti } \\
\text { Non-Kuwaiti }\end{array}$ & $\begin{array}{l}108(62.79) \\
64(37.21)\end{array}$ \\
\hline $\begin{array}{l}\text { Marital status }{ }^{d} \\
\quad \text { Married } \\
\text { Separated/divorced/widowed }\end{array}$ & $\begin{array}{r}161(93.06) \\
12(6.94)\end{array}$ \\
\hline $\begin{array}{l}\text { Educational level }{ }^{\mathrm{c}} \\
\text { No formal education } \\
\text { Primary education } \\
\text { Intermediate education } \\
\text { Secondary education } \\
\text { Diploma }\end{array}$ & $\begin{array}{c}5(2.91) \\
5(2.91) \\
16(9.30) \\
23(13.37) \\
58(33.72) \\
65(37.79)\end{array}$ \\
\hline $\begin{array}{l}\text { University degree or above } \\
\text { Total Family income in } \mathrm{KD}^{\mathrm{d}} \\
<1000 \\
1000-1499 \\
1500-1999 \\
2000+ \\
\text { Don't want to report }\end{array}$ & $\begin{array}{l}50(29.76) \\
30(17.86) \\
22(13.10) \\
44(26.19) \\
22(13.10)\end{array}$ \\
\hline \multicolumn{2}{|l|}{ Children with T1D } \\
\hline $\begin{array}{l}\text { Age, years, mean (SD) } \\
\text { Gender, Female, n (\%) }\end{array}$ & $\begin{array}{l}9.20(2.98) \\
93(53.14)\end{array}$ \\
\hline $\begin{array}{l}\text { Duration of diabetes } \\
\text { months, median (IQR) }\end{array}$ & $\begin{array}{c}32.37 \\
(18.97-54.93)\end{array}$ \\
\hline
\end{tabular}

T1D: Type 1 Diabetes, SD: Standard Deviation, KD: Kuwaiti Dinar, IQR: Interquartile range.

a Data missing on 44 participants. ${ }^{\mathrm{b}}$ Data missing on 1 participant ${ }^{\mathrm{C}}$ missing on 6 participants.

$\mathrm{d}$ missing on 7 participants. e Data missing on 34 participants.

Table 3. Difficulty of individual questions in Arabic version of the NVS (NVS-Ar).

\begin{tabular}{cc}
\hline Question number in NVS-Ar & $\begin{array}{c}\text { Correct responses } \\
\text { N (\%) }\end{array}$ \\
\hline Question 1 & $103(58.86)$ \\
Question 2 & $128(73.14)$ \\
Question 3 & $113(64.57)$ \\
Question 4 & $65(37.14)$ \\
Question 5 & $135(77.14)$ \\
Question 6 & $101(57.71)$ \\
\hline
\end{tabular}


NVS: Newest vital sign.

Table 4. Assessment of the validity of the Arabic version of the NVS (NVS-Ar): association between NVS-Ar score and the educational level of parents of children with T1D.

$\begin{array}{ccc}\begin{array}{c}\text { Limited/ possible limited } \\ \text { (score 0-3) }\end{array} & \begin{array}{c}\text { Adequate } \\ (\text { score 4-6) }\end{array} & \begin{array}{c}P \\ \text { value }\end{array} \\ (\mathrm{n}=75) & & \\ & (\mathrm{n}=100) & \end{array}$

Level of education, $n(\%)^{a}$

No formal/primary/intermediate

$12(46.15)$

$14(53.85) \quad 0.423$

Secondary school

Diploma

$13(56.52)$

$10(43.48)$

$25(43.10)$

$33(56.90)$

University degree or above

24 (36.92)

41 (63.08)

NVS: Newest vital sign, T1D: Type 1 diabetes. ${ }^{a}$ data missing from 3 participants.

\section{Figures}



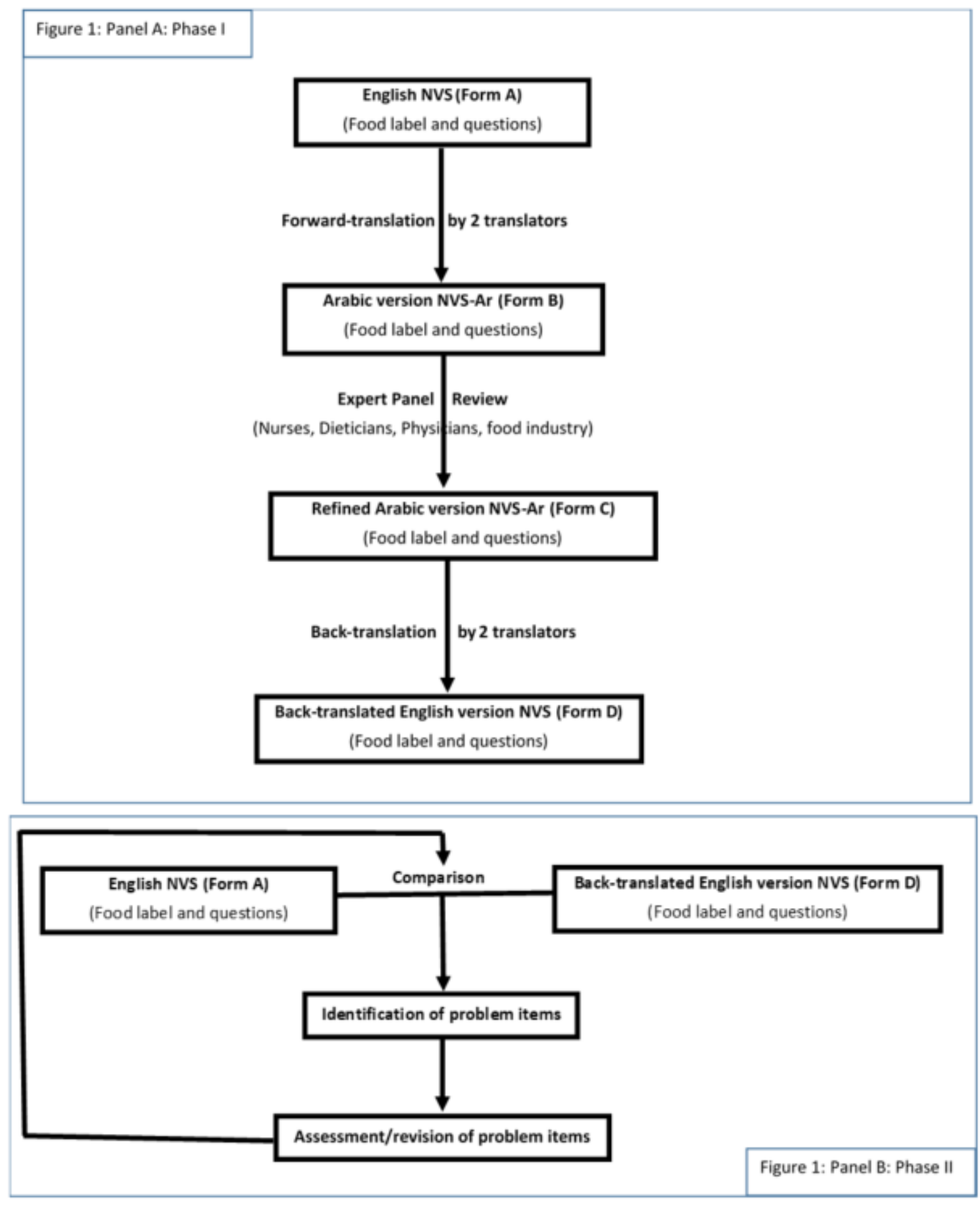

NVS: Newest vital sign.

\section{Figure 1}

Translation and Validation of the NVS tool into Arabic. 


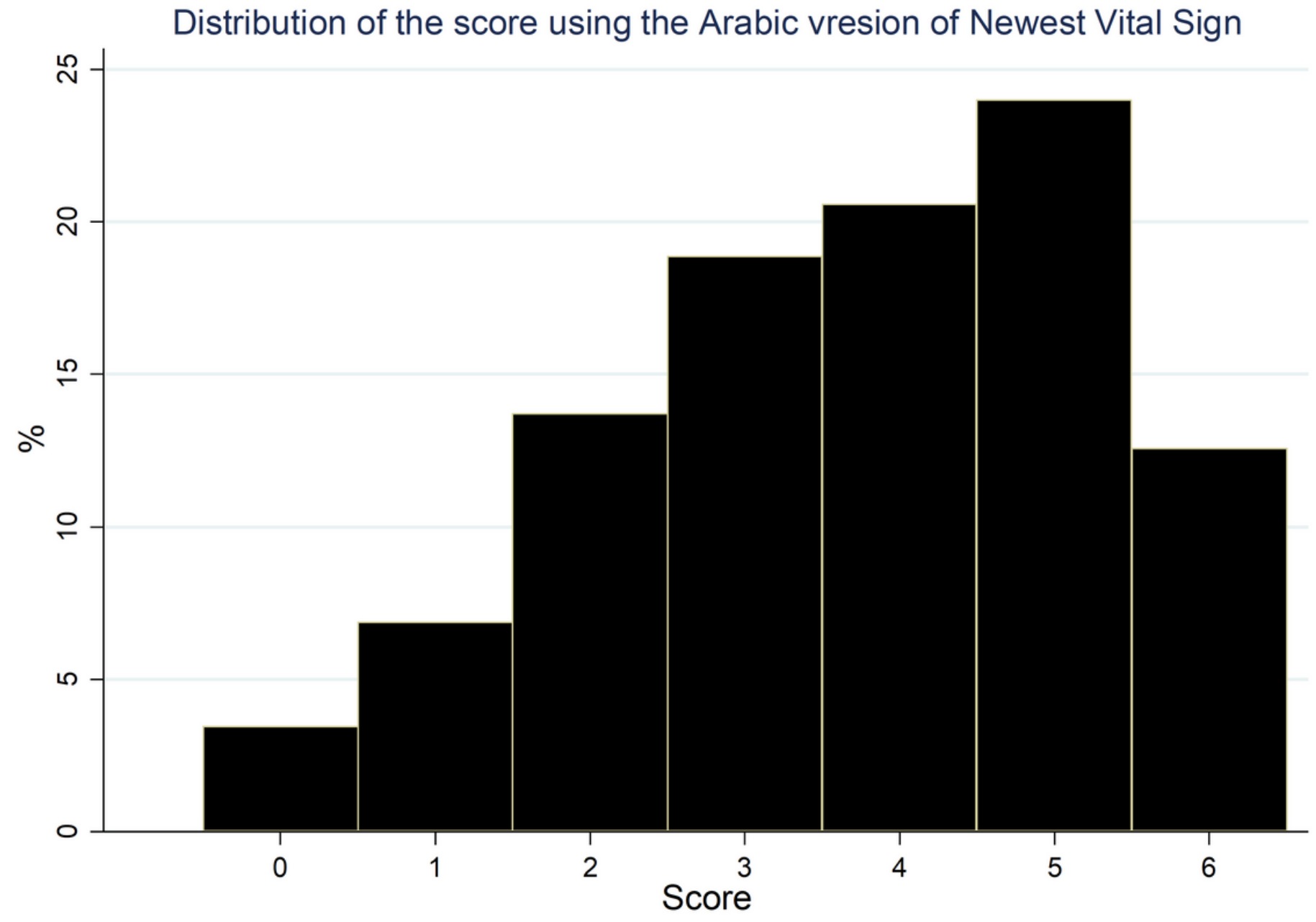

Figure 2

Distribution of total score of Newest Vital Sign in Arabic 


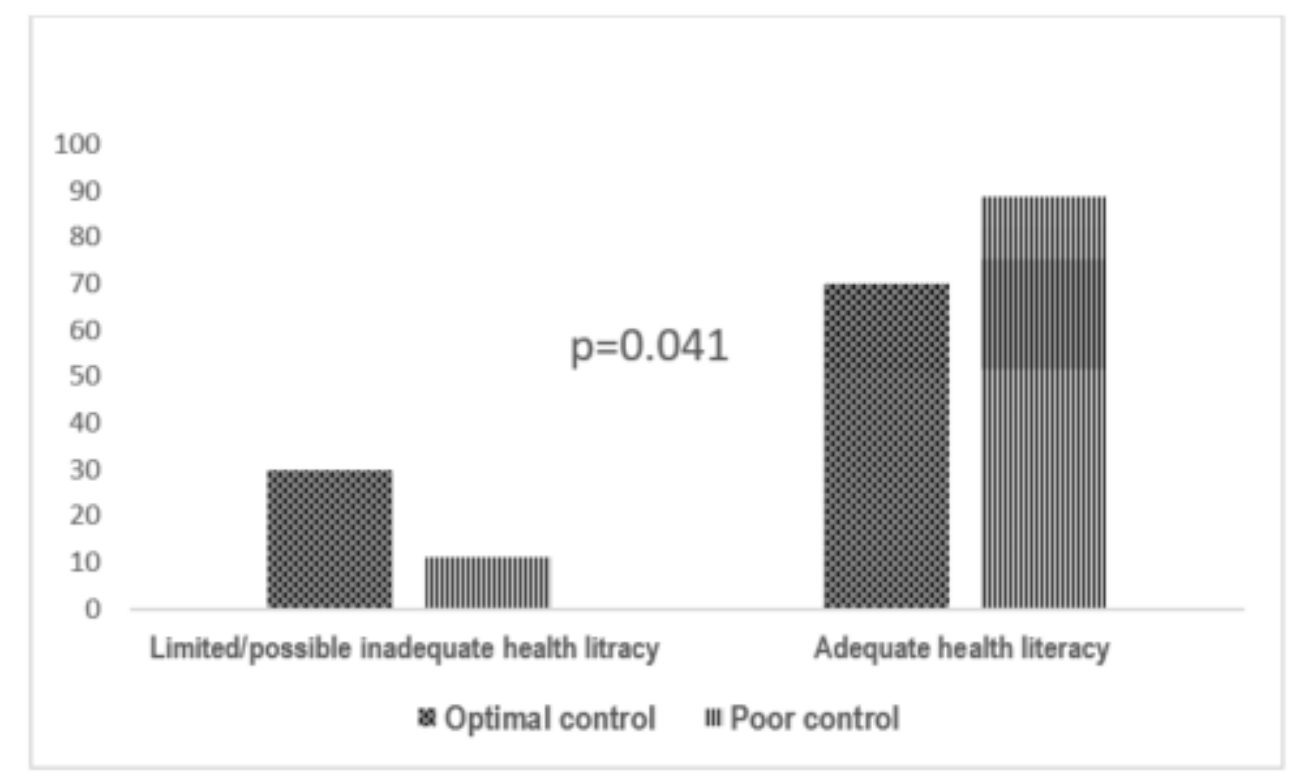

NVS: Newest vital sign, T1D: Type 1 diabetes, HbA1C: Hemoglobin A1C.

"As per the 2018 ISPAD guidelines (optimal; HbA1C $<7.5 \%$ ).

\section{Figure 3}

Assessment of the validity of the Arabic version of the NVS (NVS-Ar): association between NVS-Ar score and optimal glycemic control (HbA1C*) in 83 children with T1D. 